\title{
Preparation of Dispersed Platinum Nanoparticles on a Carbon Nanostructured Surface Using Supercritical Fluid Chemical Deposition
}

\section{Mineo Hiramatsu ${ }^{1, *}$ and Masaru Hori ${ }^{2}$}

1 Department of Electrical and Electronic Engineering, Meijo University, Tempaku, Nagoya 468-8502, Japan

2 Department of Electrical Engineering and Computer Science, Nagoya University, Chikusa, Nagoya 464-8603, Japan; E-Mail: hori@nuee.nagoya-u.ac.jp

* Author to whom correspondence should be addressed; E-Mail: mnhrmt@ccmfs.meijo-u.ac.jp; Tel.: +81-52-832-1151; Fax: +81-52-832-1298.

Received: 31 October 2009; in revised form: 30 December 2009 / Accepted: 18 January 2010 / Published: 3 March 2010

\begin{abstract}
We have developed a method of forming platinum (Pt) nanoparticles using a metal organic chemical fluid deposition (MOCFD) process employing a supercritical fluid (SCF), and have demonstrated the synthesis of dispersed Pt nanoparticles on the surfaces of carbon nanowalls (CNWs), two-dimensional carbon nanostructures, and carbon nanotubes (CNTs). By using SCF-MOCFD with supercritical carbon dioxide as a solvent of metal-organic compounds, highly dispersed Pt nanoparticles of $2 \mathrm{~nm}$ diameter were deposited on the entire surface of CNWs and CNTs. The SCF-MOCFD process proved to be effective for the synthesis of Pt nanoparticles on the entire surface of intricate carbon nanostructures with narrow interspaces.
\end{abstract}

Keywords: carbon nanostructures; supercritical fluid; platinum nanoparticles

\section{Introduction}

Graphite-related materials have long been a subject of interest to researchers. Since the first report of carbon nanotubes (CNTs) by Iijima [1], the fabrication of carbon nanostructures has been studied intensively. One-dimensional carbon nanostructures, such as carbon nanotubes and carbon nanofibers, have attracted significant interest for applications such as electrochemical devices, electron field 
emitters, hydrogen storage materials, and scanning probe microscopy, due to their favorable physical, chemical, and mechanical characteristics [2-4].

Two-dimensional carbon nanostructures have also been grown [5-7]. Layered graphene sheets can form two-dimensional carbon nanostructures with edges, called carbon nanowalls (CNWs), carbon nanosheets or carbon nanoflakes. CNWs can be described as graphite sheet nanostructures with edges composed of stacks of planar graphene sheets standing almost vertically on a substrate. The sheets form a wall structure with thicknesses in the range from a few nanometers to few tens of nanometers, and with a high aspect ratio (Figures 1 and 2). Their high aspect ratio and high surface-to-volume ratio are potentially useful as electron field emitters [8-10] and as catalyst supports [11]. It is expected that metal nanoparticles supported on the surfaces of carbon nanostructures will improve their electrical properties, and electrocatalyst/carbon nanostructure composites can be applied as electrochemical devices [11].

Figure 1. Schematic illustration of carbon nanowalls.

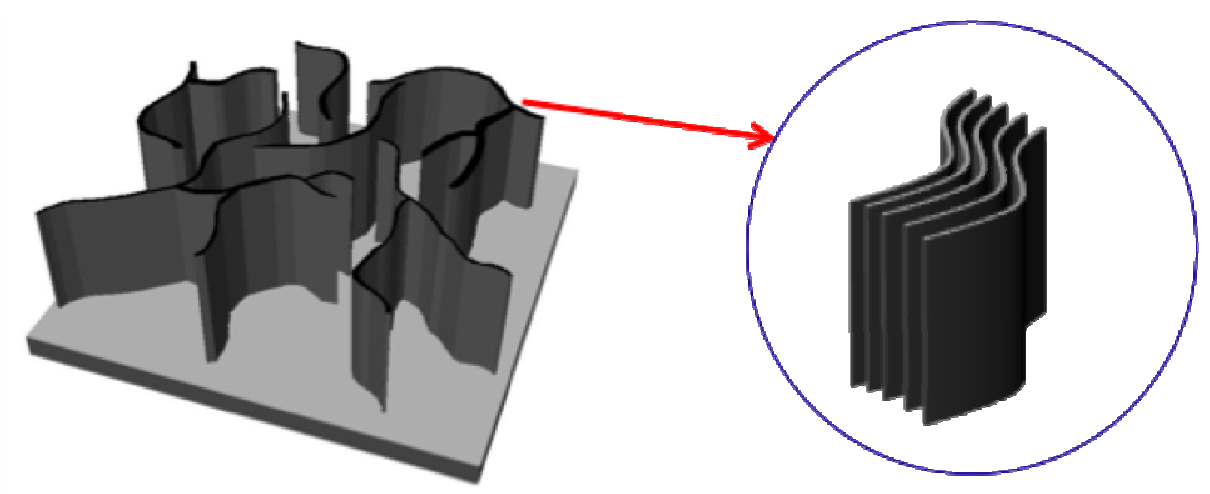

Figure 2. (a) SEM image of typical carbon nanowalls. (b) A high-resolution TEM image of a CNW, showing graphene layers of carbon nanowalls.
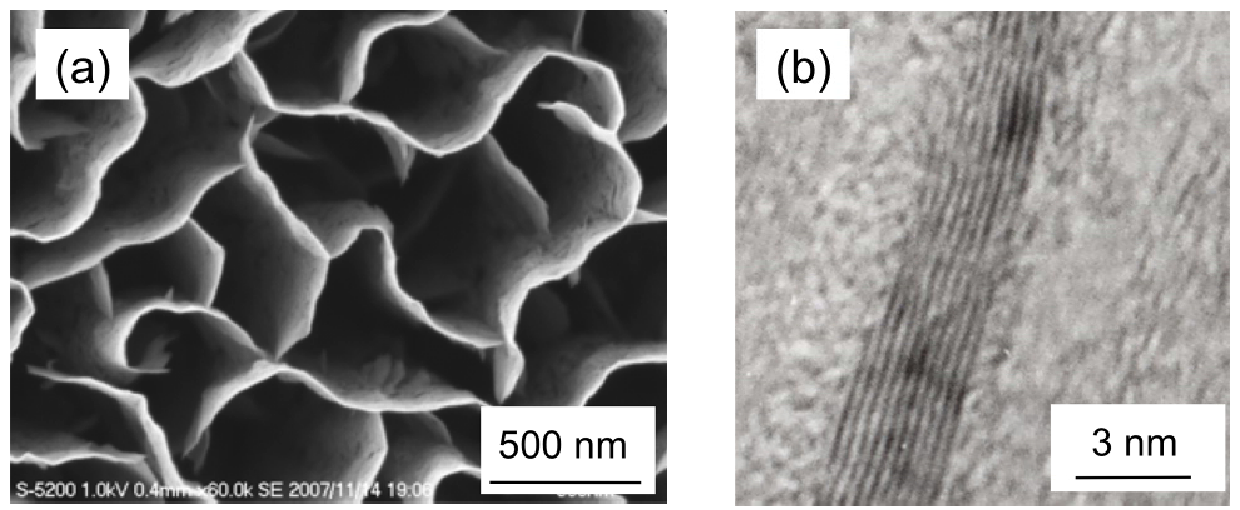

Among possible supporting materials, carbon black has been widely used as an electrode, wherein platinum (Pt) nanoparticles are dispersed [12,13]. In contrast, CNTs are considered to be a more attractive candidate due to their outstanding characteristics, including high tensile strength, large surface area, high electrical conductivity, and thermal conductivity [14-16]. Carbon nanohorns (CNH) 
and CNWs are also possible candidates for similar applications due to their large surface areas. It has been reported that CNHs can be used as Pt supports for fuel cell applications [17].

It is well known that the specific activity of catalysts is strongly related to their size, dispersion, and compatibility with supporting materials. Highly dispersed catalyst nanoparticles with small size and narrow size distribution supported on carbon nanostructures are ideal for high electrocatalyst activity due to their large surface-to-volume ratio. To support metal nanoparticles on the surface of carbon nanostructures, metal compounds in the form of liquids are generally employed. A few papers have been published on the preparation of Pt nanoparticles on CNT surfaces by the reduction of Pt salt precursors such as $\mathrm{H}_{2} \mathrm{PtCl}_{6}$ in solution $[18,19]$. On the other hand, it is difficult to treat the entire surface of CNWs with a metal compound in a liquid phase, because of the high surface tension of CNWs due to their high aspect ratio with narrow interspaces. Therefore, there is an urgent need for new methods of metal nanoparticle deposition on carbon nanostructures. One promising method is to employ gas phase deposition, such as sputtering and chemical vapor deposition. However, in gas phase deposition, metal nanoparticles are deposited only around the tops of CNWs and tend to easily clump together, resulting in the formation of larger particles or films on the top of carbon nanostructures.

As an alternative approach to support metal nanoparticles on the surfaces of dense, aligned CNTs and CNWs with narrow interspaces, we demonstrate a method employing metal-organic chemical fluid deposition (MOCFD), where supercritical carbon dioxide $\left(\mathrm{sc}-\mathrm{CO}_{2}\right)$ is used as a solvent of metalorganic compounds. The supercritical fluid (SCF) possesses attractive properties of both the gas and the liquid phases. Rapid diffusion and permeation are realized by its gas-like diffusivity and viscosity, while its liquid-like density enables dissolution of a wide range of materials. To produce an SCF phase, the temperature and pressure of the material are required to exceed the critical point. The critical point of sc- $\mathrm{CO}_{2}$ exists at $7.38 \mathrm{MPa}(72.8 \mathrm{~atm})$ and $31.1{ }^{\circ} \mathrm{C}$. Among $\mathrm{SCFs}, \mathrm{sc}-\mathrm{CO}_{2}$ is particularly attractive since it is environmentally friendly and safe due to its low toxicity, low reactivity and nonflammability.

Cansell and Aymonir have surveyed research on the synthesis of functional nanostructured materials utilizing the specific properties of SCFs over the past five years [20]. Recent advances in the synthesis of CNT composites using SCFs have been reviewed by Liu and Han, with emphasis on metal/CNT, metal-oxide/CNT, and polymer/CNT composites [21]. In the case of Pt deposition, the SCF using sc- $\mathrm{CO}_{2}$ was first applied to the preparation of polymer-supported Pt nanoparticles using dimethyl(1,5-cyclooctadiene) platinum(II), $\left(\mathrm{PtMe}_{2}(\mathrm{cod})\right)$ as a precursor [22]. Pt nanoparticles of 5-15 nm size were prepared on the CNT surface by hydrogen reduction of $\mathrm{Pt}(11)$ acetylacetonate, $\left(\mathrm{Pt}(\mathrm{acac})_{2}\right)$ in methanol-modified $\mathrm{sc}-\mathrm{CO}_{2}$ [23]. Erkey's group has demonstrated the preparation of $\mathrm{Pt}$ nanoparticles on a wide range of materials, including carbon aerogel, carbon black, silica aerogel, alumina, and Nafion [24-27]. In their method, Pt nanoparticles were prepared by impregnating $\mathrm{PtMe}_{2}$ (cod) into the substrates from $\mathrm{sc}-\mathrm{CO}_{2}$ solution for $\sim 6$ hours. After depressurization, the impregnated metal-organic compound was reduced to elemental Pt by heat treatment in the presence of nitrogen gas for $\sim 4$ hours. It took almost 10 hours to complete the process for the preparation of Pt nanoparticles.

In this work, we have developed a method of formation of Pt nanoparticles using the MOCFD process employing sc- $\mathrm{CO}_{2}$ as a solvent of metal-organic compounds, and have demonstrated the synthesis of highly dispersed $\mathrm{Pt}$ nanoparticles of $2 \mathrm{~nm}$ size without heat treatment after 
depressurization, on the entire surface of two kinds of carbon nanostructures, CNWs with high aspect ratio and narrow interspaces and aligned CNTs $[28,29]$.

\section{Experimental}

Figure 3 shows the SCF-MOCFD system employing sc- $\mathrm{CO}_{2}$ used for the deposition of $\mathrm{Pt}$ nanoparticles on the surface of CNW and CNT samples [28,29]. The MOCFD process was conducted in two high-pressure stainless steel vessels equipped with a compressor, heating units, and a reservoir for the metal-organic compound. The preliminary vessel contains a screw agitator. The temperature and pressure in each vessel can be set independently, so that two different supercritical conditions employing $\mathrm{CO}_{2}$ can be produced in these two vessels. As the precursor, we used (methylcyclopentadienyl) trimethyl platinum $\left(\left(\mathrm{CH}_{3} \mathrm{C}_{5} \mathrm{H}_{4}\right) \mathrm{Pt}\left(\mathrm{CH}_{3}\right)_{3}: \mathrm{MeCpPtMe}_{3}\right)$ dissolved in hexane. The concentration of $\mathrm{MeCpPtMe}_{3}$ was $1 \mathrm{wt} \%$, and the quantity of the solution used was $5 \mathrm{~mL}$. In the preliminary vessel, the precursor was stirred with the $\mathrm{sc}-\mathrm{CO}_{2}$ for about $30 \mathrm{~min}$ to realize high diffusion of the metal-organic compound in the sc- $\mathrm{CO}_{2}$. In the impregnation vessel, the selective heating of CNW or CNT samples during the MOCFD process facilitated selective metal deposition on the surface of the carbon nanostructures. With help of the needle valve connecting these vessels, it is possible to mix two different fluids and start the metal nanoparticle deposition. In the preliminary vessel, the pressure and temperature of $\mathrm{sc}-\mathrm{CO}_{2}$ were maintained at $11 \mathrm{MPa}$ and $50{ }^{\circ} \mathrm{C}$, respectively, and $\mathrm{MeCpPtMe}_{3}$ was dissolved in the sc- $\mathrm{CO}_{2}$. In the impregnation vessel, the pressure and temperature of sc- $\mathrm{CO}_{2}$ were maintained at $9 \mathrm{MPa}$ and $70{ }^{\circ} \mathrm{C}$, respectively, and the temperature of $\mathrm{CNW}$ or $\mathrm{CNT}$ samples was controlled in the range of $70-170{ }^{\circ} \mathrm{C}$. Finally, the solutions were mixed and $\mathrm{Pt}$ nanoparticles formation was carried out for $30 \mathrm{~min}$; the vessel was then depressurized slowly in $30 \mathrm{~min}$ to atmospheric conditions. After the depressurization, no additional heat treatment was carried out in the present work.

Figure 3. Schematic of the supercritical fluid, metal-organic chemical fluid deposition (SCF-MOCFD) system used in this study [28,29].

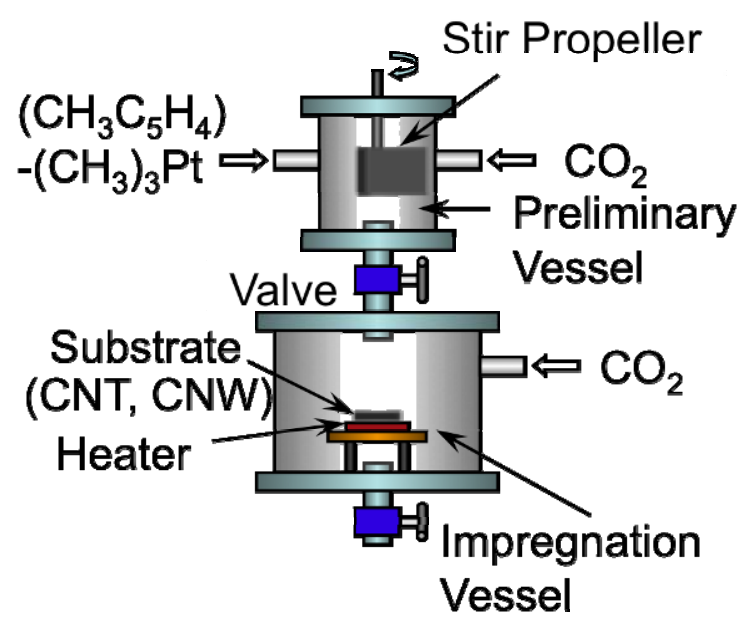

CNW samples were fabricated on a Si substrate by fluorocarbon $\left(\mathrm{C}_{2} \mathrm{~F}_{6}\right)$ plasma enhanced chemical vapor deposition assisted by hydrogen radical injection, comprised of a parallel-plate very high frequency (VHF: $100 \mathrm{MHz}$ ), capacitively coupled plasma region, and a hydrogen radical injection 
source that employs a surface-wave-excited microwave $(2.45 \mathrm{GHz}) \mathrm{H}_{2}$ plasma [30]. The average height of a typical CNW film sample used in this study was approximately $900 \mathrm{~nm}$, while the thickness of each nanowall was less than $10 \mathrm{~nm}$. Meanwhile, aligned CNT film samples were prepared on the Si substrates using microwave plasma enhanced chemical vapor deposition with a $2.45 \mathrm{GHz}, 1.5 \mathrm{~kW}$ microwave generator. Details of the synthesis method of the CNT films are described elsewhere [31,32]. A mixture of $\mathrm{CH}_{4}$ and $\mathrm{H}_{2}$ was used as the source gas. By the controlled preparation of catalytic Co nanoparticles, vertically aligned, single-walled, and double-walled CNT films were fabricated in a controlled manner.

Scanning electron microscopy (SEM) and transmission electron microscopy (TEM) were used to characterize the morphology of the carbon nanostructures and the Pt particle size supported on the surface of the CNWs and CNTs. The presence of Pt was detected by energy dispersive X-ray spectrometry (EDX) and X-ray photoelectron spectroscopy (XPS).

\section{Results}

Figure 4 shows a cross-sectional SEM image of a CNW film after the plating treatment, together with a magnified SEM top view image of the CNWs in the inset. By using the plating method, $\mathrm{Pt}$ nanoparticles $(5-10 \mathrm{~nm})$ were deposited only on the tops of the CNWs, as shown in the inset in Figure 4. Figures 5(a) and 5(b) show a cross-sectional SEM image and a low-resolution TEM image of CNW film after the sputtering treatment, respectively. Magnified TEM images of CNWs near the top and middle positions are shown in Figures 5(c) and 5(d). In this case, Pt nanoparticles (2-3 nm) were deposited near the top of the CNW film. From the results shown in Figures 4 and 5, it is seen that the preparation of Pt nanoparticles on the entire surface of CNWs could not be realized by deposition methods using the liquid phase or the gas phase.

Figure 4. A cross-sectional SEM of a carbon nanowall film after the plating treatment, together with a magnified SEM top view image of the carbon nanowalls in the inset.

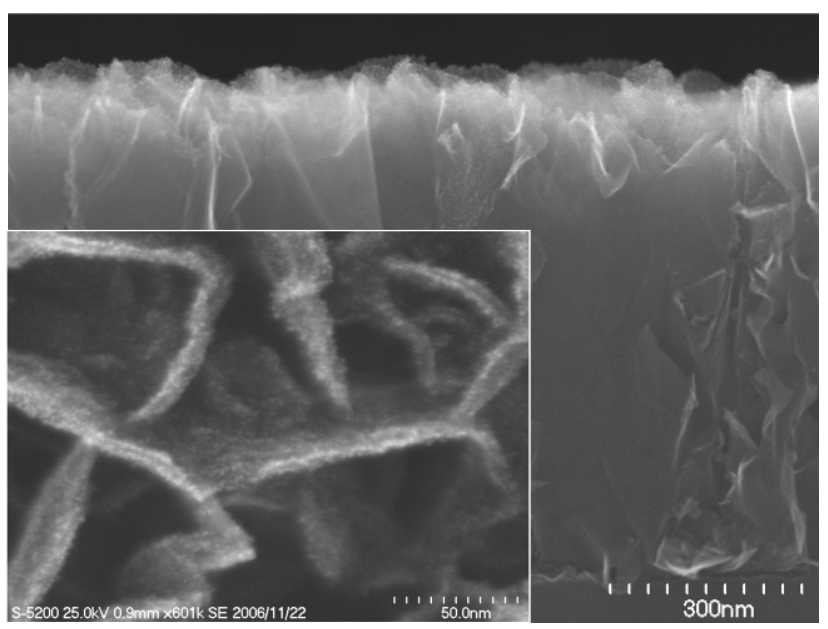


Figure 5. (a) A cross-sectional SEM image of the CNW film, and (b) a low-resolution TEM image of the CNW film after the sputtering treatment. (c) TEM image of the CNW film at the "top" position in the cross-sectional TEM image in Figure 5(b). (d) TEM image of the CNW film at the "middle" position in the cross-sectional TEM image in Figure 5(b) [28].
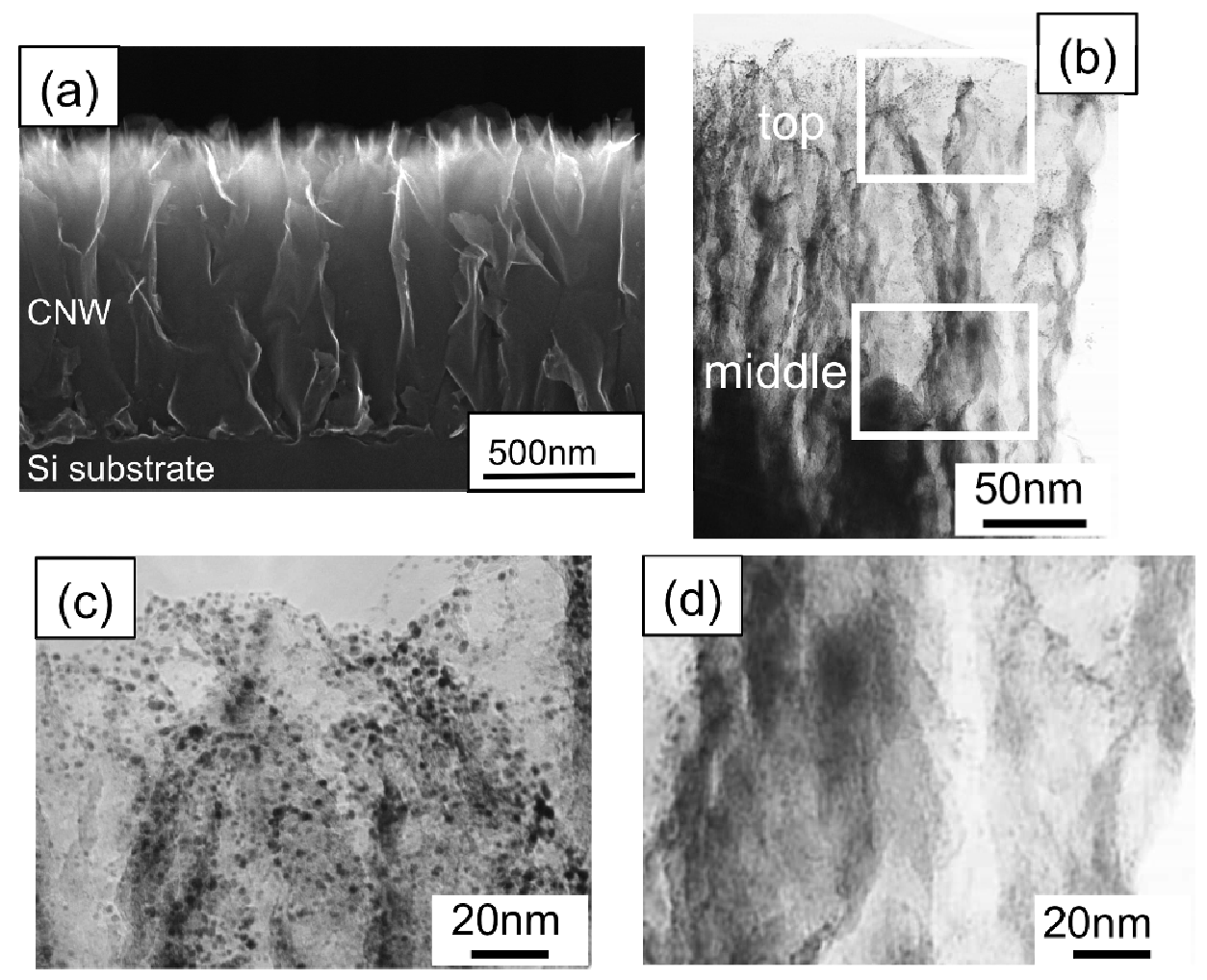

Figure 6(a) shows an SEM top-view image of a CNW film sample after the SCF-MOCFD treatment at a sample temperature of $150{ }^{\circ} \mathrm{C}$. Compared to the SEM images of typical as-grown $\mathrm{CNW}$ films without the SCF treatment (data not shown), no change in the surface morphology was observed. It was confirmed that the unique nanostructure of the CNWs was maintained, even after being exposed to the high-pressure fluid. Figure 6(b) shows a low-resolution TEM image of detached CNWs after the SCF-MOCFD treatment. Nanoparticles are supported on the entire surface of the CNWs. A magnified TEM image of the surface of the CNW after the SCF-MOCFD is shown in the inset in Figure 6(b), indicating the presence of dispersed nanoparticles of approximately $2 \mathrm{~nm}$ size on the CNW surface. These nanoparticles have also been identified to be pure platinum by EDX analysis. Figure 6(c) shows a high-resolution TEM image of the surface of the CNW supporting the Pt nanoparticles, and $0.23 \mathrm{~nm}$ spaced lattice fringes corresponding to the $d_{111}$ interplanar distance of platinum, well-oriented to the electron beam are observed. The high-resolution TEM image shown in Figure 6(c) also clearly reveals the graphene layers of the CNWs, indicating the graphitized structure of the CNWs. The spacing between neighboring graphene layers was measured to be approximately $0.34 \mathrm{~nm}$. 
Figure 6. (a) SEM top-view image of a Pt-supported CNW film after SCF-MOCFD treatment at a sample temperature of $150{ }^{\circ} \mathrm{C}$. (b) Low-resolution TEM image of detached CNWs after the SCF-MOCFD treatment. Inset: magnified TEM image of the surface of the CNW supporting Pt nanoparticles. (c) High resolution TEM image showing the $\mathrm{d}_{111} \mathrm{Pt}$ interplanar distance and graphene layers of the CNW [29].
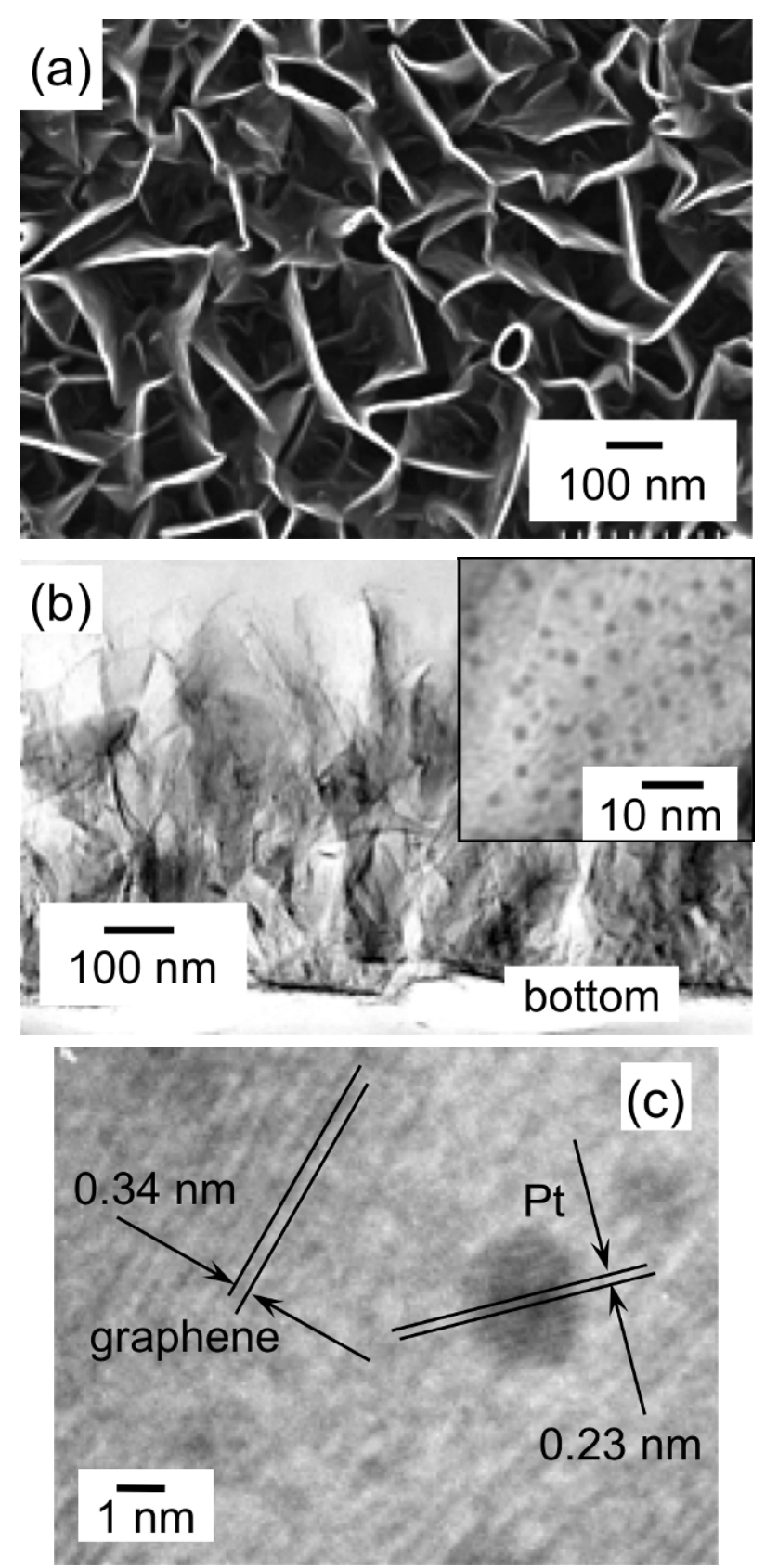

Figure 7 shows the distribution histogram of the Pt nanoparticle size formed at $150{ }^{\circ} \mathrm{C}$ deduced from TEM observations, indicating that the Pt nanoparticles supported on the CNW surface had a small average size and a narrow size distribution centered at approximately $2-2.5 \mathrm{~nm}$. 
Figure 7. Distribution histogram of Pt nanoparticle size supported on the CNW surface at $150{ }^{\circ} \mathrm{C}[29]$.

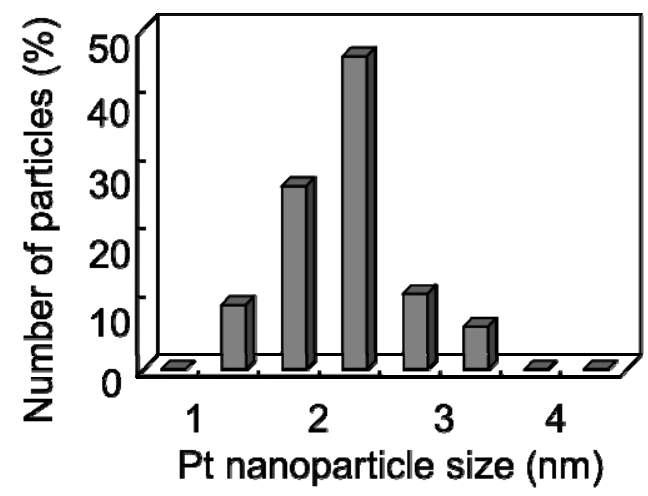

Ex situ XPS analysis was conducted to gain an insight into the state of the platinum in the supported Pt surface fabricated by the SCF-MOCFD method. Figure 8(a) shows an XPS spectrum of the Pt (4f) region of the Pt-supported CNW film after the SCF-MOCFD at a sample temperature of $150{ }^{\circ} \mathrm{C}$. For comparison, Figure 8(b) shows an XPS spectrum of Pt particles deposited on the CNW film after the plating treatment shown in Figure 4. The presence of two prominent sets of $\mathrm{Pt}(4 f)$ peaks, corresponding to the $4 f_{7 / 2}$ and $4 f_{5 / 2}$ orbital states, is further confirmation of platinum being present on the CNW surface. The peak regions in Figure 8(a) can be fitted with two sets of peaks at $71.4 \mathrm{eV}$ $\left(4 f_{7 / 2}\right)$ and $74.6 \mathrm{eV}\left(4 f_{5 / 2}\right)$ [33]. These correspond to platinum in the metallic state, indicating that only pure Pt exists without being oxidized on the surface of the CNWs after the SCF-MOCFD. On the other hand, in the case of the XPS spectrum measured for the Pt particles deposited on the CNW film after the plating treatment shown in Figure 8(b), the peak regions can be fitted with two sets of peaks at 71.4 and $74.6 \mathrm{eV}$ corresponding to platinum in the metallic state, and shoulder peaks at 72.4 and $76.5 \mathrm{eV}$. These shoulder peaks correspond to platinum in an oxide form [33]. Thus, in the case of deposition by plating, platinum on the surface of the $\mathrm{CNW}$ film is present in elemental as well as oxide states.

Figure 8. X-ray photoelectron spectroscopy spectra of the Pt-supported CNW film after (a) SCF-MOCFD and (b) plating [29].
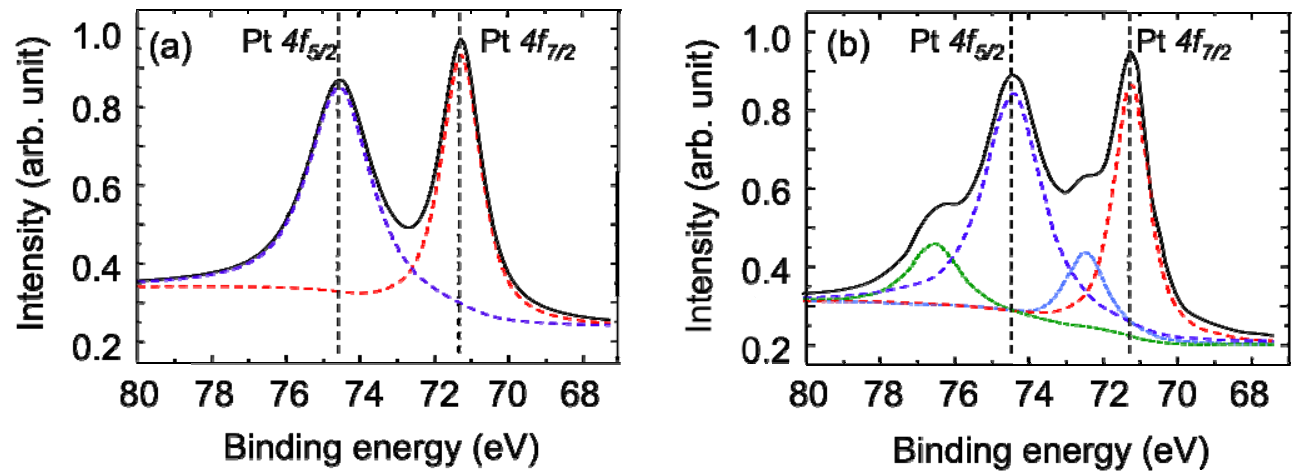

The $\mathrm{Pt} / \mathrm{C}$ ratio of the $\mathrm{CNW}$ film surface was obtained from the ratio of the intensities of the XPS C $(1 s)$ and $\mathrm{Pt}(4 f)$ peaks. Figure 9 (a) shows the relative $\mathrm{Pt} / \mathrm{C}$ ratio of the surface of the $\mathrm{CNW}$ film as a function of the temperature of the CNW sample during the SCF-MOCFD process. As the sample 
temperature during the SCF-MOCFD increased up to $120^{\circ} \mathrm{C}$, the relative $\mathrm{Pt} / \mathrm{C}$ ratio of the surface of the $\mathrm{CNW}$ film increased gradually. By further increasing the sample temperature above $120{ }^{\circ} \mathrm{C}$, the relative $\mathrm{Pt} / \mathrm{C}$ ratio increased rapidly. Figures 9(b), 9(c), and 9(d) show TEM images of the Pt-supported CNW surface after the SCF-MOCFD at sample temperatures of 120,150 , and $170{ }^{\circ} \mathrm{C}$, respectively. As can be seen from these TEM images, the spatial density of the Pt nanoparticles (particle numbers/area) supported on the CNW surface strongly depended on the sample temperature during the SCF-MOCFD, while the average size of the Pt nanoparticles increased from 1.5 to $3 \mathrm{~nm}$ with an increase in the sample temperature from 120 to $170{ }^{\circ} \mathrm{C}$.

Figure 9. (a) Relative $\mathrm{Pt} / \mathrm{C}$ ratio of the surface of the $\mathrm{CNW}$ film as a function of temperature of the CNW sample during SCF-MOCFD. (b)-(d) High-resolution TEM images of the surface of the CNW supporting Pt nanoparticles after the SCF-MOCFD at sample temperatures of 120,150 , and $170{ }^{\circ} \mathrm{C}$, respectively [29].
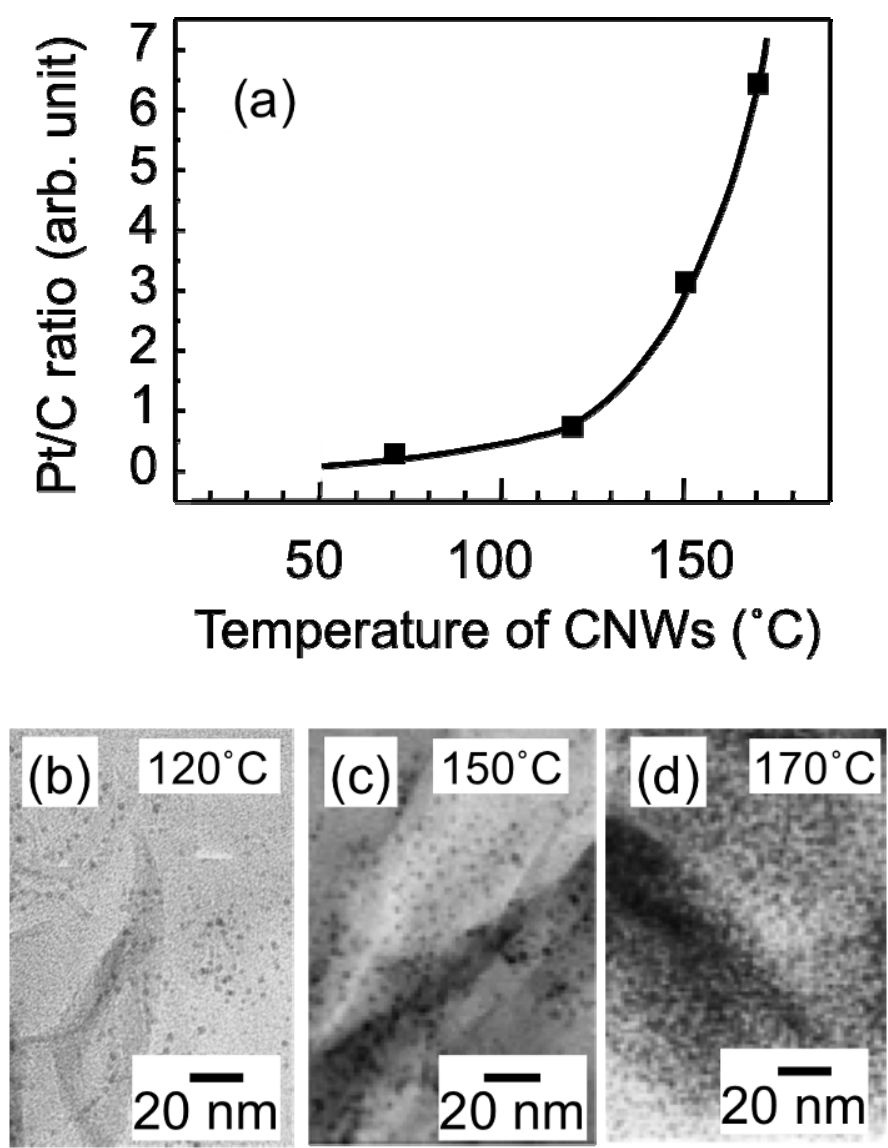

A TEM image of CNTs scraped away from the substrate before the SCF-MOCFD treatment is shown in Figure 10(a), together with the cross-sectional SEM image of a cleaved, aligned CNT film as an inset. The TEM image shown in Figure 10(a) reveals that most of the CNTs are double-walled, with an average outer diameter of approximately $4 \mathrm{~nm}$. These vertically aligned, dense CNT films were exposed to the $\mathrm{sc}-\mathrm{CO}_{2}$ with $\mathrm{MeCpPtMe}_{3}$. TEM images of the CNT film after the SCF-CVD are shown in Figures 10(b)-10(d). Figure 10(b) shows a low-resolution cross-sectional TEM image of the Pt-supported CNT film after the SCF-MOCFD treatment at a sample temperature of $150{ }^{\circ} \mathrm{C}$. The TEM 
image in Figure 10(c) corresponds to the position indicated as "top" in the cross-sectional TEM image of the CNT film shown in Figure 10(b), while the TEM image in Figure 10(d) corresponds to the "middle" position. The TEM images observed at both positions indicate that the entire surface of the CNTs was covered with platinum. Figure 10(e) shows a TEM image of the CNTs scraped away from the substrate after the SCF-MOCFD treatment at a sample temperature of $150{ }^{\circ} \mathrm{C}$. As a result of the SCF-MOCFD, metal nanoparticles were formed on the entire surface of the CNTs. Figure 10(f) shows a magnified TEM image of double-walled CNTs after the SCF-MOCFD, indicating the presence of dispersed nanoparticles of approximately $2 \mathrm{~nm}$ size on the CNT surface.

Figure 10. (a) TEM image of CNTs scraped away from the substrate before SCF-MOCFD treatment. Inset: cross-sectional SEM image of a cleaved aligned CNT film grown using microwave plasma-enhanced chemical vapor deposition. (b) Low-resolution crosssectional TEM image of a Pt-supported CNT film after the SCF-MOCFD treatment at a sample temperature of $150{ }^{\circ} \mathrm{C}$, (c) TEM image of Pt-supported CNT film at the "top" position in the cross-sectional TEM image of Figure 10(b), and (d) TEM image of Ptsupported CNT film at the "middle" position in the cross-sectional TEM image of Figure 10(b). (e) TEM image of CNTs scraped from the substrate after the SCF-MOCFD treatment at a sample temperature of $150{ }^{\circ} \mathrm{C}$. (f) Magnified TEM image of double-walled CNTs scraped from the substrate after the SCF-MOCFD, indicating the presence of dispersed nanoparticles on the CNT surface [29].
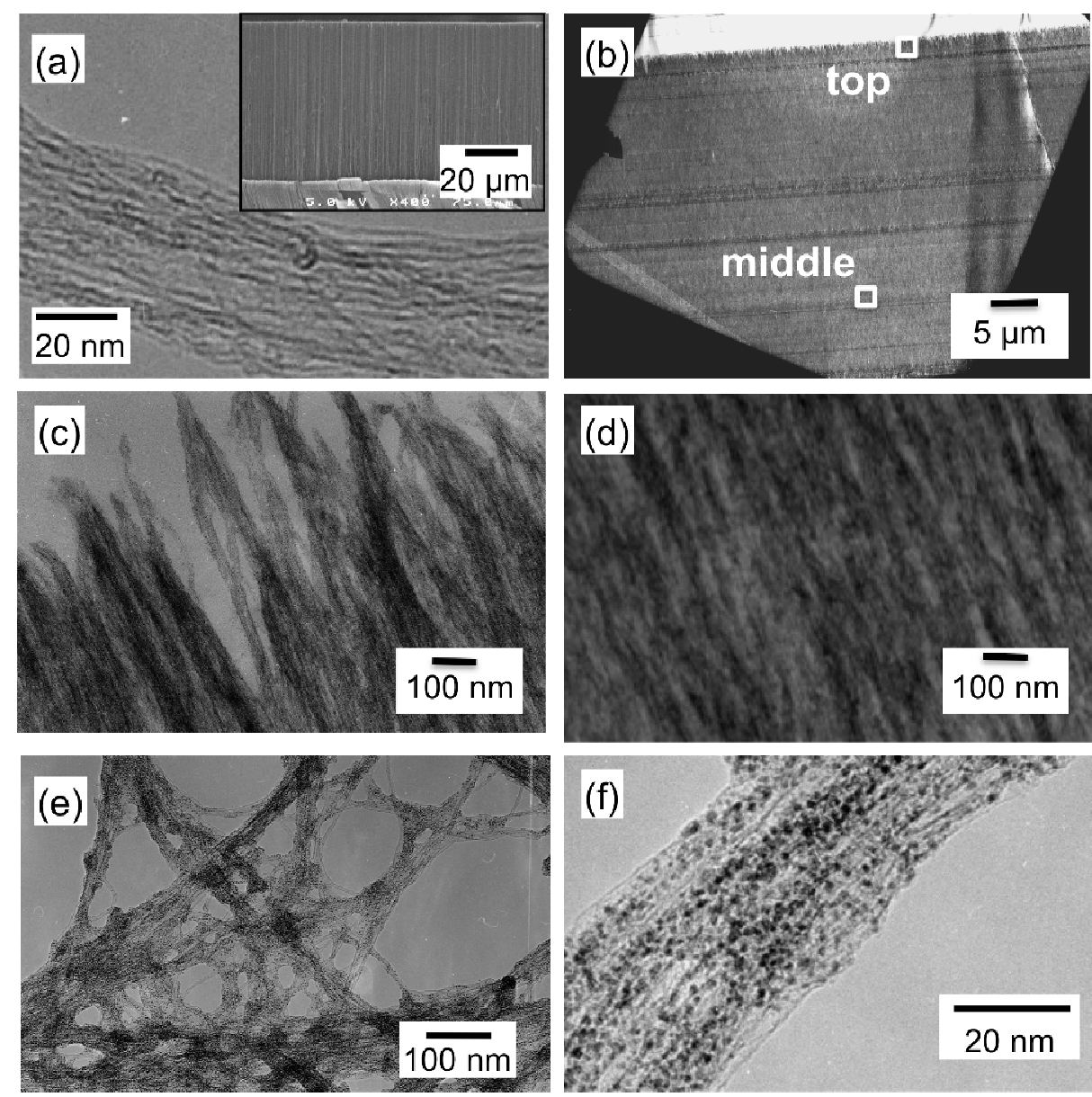


\section{Discussion}

$\mathrm{MeCpPtMe}_{3}$ has been used as a precursor for the formation of Pt particles and films by metalorganic chemical vapor deposition (MOCVD) for years. Thermal decomposition of $\mathrm{MeCpPtMe}_{3}$ has been reported to occur at $240{ }^{\circ} \mathrm{C}$, while the initial crystal growth of platinum from the precursors on a highly oriented pyrolytic graphite was observed at $190{ }^{\circ} \mathrm{C}$ [34]. In contrast, in our case using the SCF-MOCFD method, the formation of Pt nanoparticles on the surface of carbon nanostructures occurred at relatively low temperatures above $120{ }^{\circ} \mathrm{C}$ as shown in Figure 9(a). In an earlier study carried out by Watkins and McCarthy [22], $\mathrm{PtMe}_{2}(\mathrm{cod})$ was converted to metallic platinum at $140{ }^{\circ} \mathrm{C}$, 26.5 $\mathrm{MPa}$ in sc- $\mathrm{CO}_{2}$, while the decomposition temperature of $\mathrm{PtMe}_{2}(\mathrm{cod})$ is $208{ }^{\circ} \mathrm{C}$ at atmospheric pressure, which is consistent with our result using the $\mathrm{MeCpPtMe}_{3}$ precursor. In the presence of a high-pressure SCF, the metal-organic precursor can be converted to elemental metal at a lower temperature than at atmospheric pressure.

Erkey's group investigated the particle formation mechanism for a variety of metals using sc- $\mathrm{CO}_{2}$ deposition [26,35]. In their method, $\mathrm{PtMe}_{2}(\mathrm{cod})$ was dissolved in $\mathrm{sc}-\mathrm{CO}_{2}$ and impregnated into the supporting materials, and after depressurization the impregnated $\mathrm{PtMe}_{2}(\mathrm{cod})$ molecules were then reduced to metallic Pt nanoparticles by heat treatment or by chemical reduction with hydrogen, resulting in the formation of uniformly dispersed nanoparticles with narrow size distributions. They proposed that the precursor molecules in the sc- $\mathrm{CO}_{2}$ phase are adsorbed on the surface, and after depressurization these adsorbed molecules are in turn reduced to elemental platinum and the resulting particles at the surface continue to grow until all the adsorbed precursor molecules are converted to the metal. When the reduction temperature of $\mathrm{PtMe}_{2}(\mathrm{cod})$ molecules was increased, the resulting particle size increased, due to the increase in mobility of the individual Pt particles, allowing them to coalesce and form larger particles.

In our case, in contrast, the supporting carbon nanostructures were selectively heated in the sc- $\mathrm{CO}_{2}$ with Pt precursors during the process. Therefore, at the heated surface of the carbon nanostructures during in situ thermal reduction under the SCF environment, decomposition of the adsorbed precursor molecules and growth of the particles would occur. Many defects exist on the surface of CNTs grown by plasma-enhanced chemical vapor deposition. Meanwhile, the CNWs have been reported to consist of nanodomains a few tens of nanometers in size [36], and individual CNWs were found to have many defects [37]. It is suggested that the surface-migrating Pt adatoms produced by the decomposition of $\mathrm{MeCpPtMe}_{3}$ precursors merge to form Pt clusters from several Pt atoms preferentially at chemically active sites such as defects and grain boundaries on the surface of the carbon nanostructures, resulting in the nucleation of Pt nanoparticles.

The reaction temperature at the surface would be a significant factor influencing the particle number density and particle size. As pointed out by Erkey's group [26], when the temperature is increased, both reduction of metal-organic precursors and surface migration of Pt atoms would be enhanced, which may lead to an increase in the particle number density and particle size. As can be seen from the TEM images in Figures 9(b)-9(d), the average size of Pt nanoparticles increased from 1.5 to $3 \mathrm{~nm}$ with an increase in the sample temperature from 120 to $170{ }^{\circ} \mathrm{C}$, while the $\mathrm{Pt}$ particle number density increased drastically. The amount of Pt loading supported on the CNW surface has not been measured. Under the constant period of the SCF-MOCFD process, the amount of precursors 
arriving at the CNW surface is assumed to be always almost the same, in spite of differences in the sample temperatures. Assuming that sufficient amount of precursors arrive at the surface, the Pt nucleation density (nucleation sites/area) would be determined by the rate of reduction of precursor molecules on the surface, which would be enhanced by increasing the surface temperature. Therefore, the amount of Pt loading is considered to increase with an increase in the sample temperature, as can be expected from the TEM images shown in Figures 9(b)-9(d). Furthermore, with regards to the growth experiment in our study, the processing period including impregnation and reduction was only $30 \mathrm{~min}$, considerably shorter than the typical period of other groups and was not sufficient for aggregation of particles. This could explain the small particle size and size distribution in this study. The large surface area of carbon nanostructures to the relevant precursor amount in the system can also account for the small particle size. If the SCF-MOCFD process is carried out at high surface temperatures for a long period ( hours), the size of the Pt particles would increase and aggregation would likely occur.

\section{Conclusions}

We have developed a new method of deposition using supercritical carbon dioxide to treat the entire surface of carbon nanostructures. We demonstrated the synthesis of dispersed Pt nanoparticles using metal-organic chemical fluid deposition employing the supercritical fluid (SCF-MOCFD). The proposed SCF-MOCFD method proved quite effective for the synthesis of Pt nanoparticles on the entire surface of aligned carbon nanotubes and carbon nanowalls with narrow interspaces. The size of the Pt nanoparticles synthesized at $150^{\circ} \mathrm{C}$ was approximately $2 \mathrm{~nm}$.

\section{References}

1. Iijima, S. Helical microtubules of graphitic carbon. Nature 1991, 354, 56-58.

2. Rinzler, A.G.; Hafner, J.H.; Nikolaev, P.; Lou, L.; Kim, S.G.; Tomanek, D.; Nordlander, P.; Cobert, D.T.; Smalley, R.E. Unraveling nanotubes: Field emission from an atomic wire. Science 1995, 269, 1550-1553.

3. Dillon, A.C.; Jones, K.M.; Bekkedahl, T.A.; Kiang, C.H.; Bethune, D.S.; Heben, M.J. Storage of hydrogen in single walled carbon nanotubes. Nature 1997, 386, 377-379.

4. Che, G.; Lakshmi, B.B.; Fisher, E.R.; Martin, C.R. Carbon nanotubule membranes and possible applications to electrochemical energy storage and production. Nature 1998, 393, 346-347.

5. Wu, Y.H.; Qiao, P.W.; Chong, T.C.; Shen, Z.X. Carbon nanowalls grown by microwave plasma enhanced chemical vapor deposition. Adv. Master. 2002, 14, 64-67.

6. Hiramatsu, M.; Shiji, K.; Amano, H.; Hori, M. Fabrication of vertically aligned carbon nanowalls using capacitively coupled plasma-enhanced chemical vapor deposition assisted by hydrogen radical injection. Appl. Phys. Lett. 2004, 84, 4708-4710.

7. Wang, J.J.; Zhu, M.Y.; Outlaw, R.A.; Zhao, X.: Manos, D.M.; Holloway, B.C.; Mammana, V.P. Free-standing subnanometer graphite sheets. Appl. Phys. Lett. 2004, 85, 1265-1267.

8. Hiramatsu, M.; Hori, M. Fabrication of carbon nanowalls using novel plasma processing. Jpn. J. Appl. Phys. 2006, 45, 5522-5527. 
9. Chuang, A.T.H.; Robertson, J.; Boskovic, B.O.; Koziol, K.K.K. Three-dimensional carbon nanowall structures. Appl. Phys. Lett. 2007, 90, 123107:1-123107:3.

10. Hou, K.; Outlaw, R.A.; Wang, S.; Zhu, M.; Quinlan, R.A.; Manos, D.M.; Kordesch, M.E.; Arp, U.; Holloway, B.C. Uniform and enhanced field emission from chromium oxide coated carbon nanosheets. Appl. Phys. Lett. 2008, 92, 133112:1-133112:3.

11. Yang, B.J.; Wu, Y.H.; Zong, B.Y.; Shen, Z.X. Electrochemical synthesis and characterization of magnetic nanoparticles on carbon nanowall templates. Nano Lett. 2002, 2, 751-754.

12. Lamy, C.; Leger, J.-M.; Srinivasan, S.M. Direct methanol fuel cells: from a twentieth century electrochemist's dream to a twenty-first century emerging technology. In Modern Aspects of Electrochemistry; O'Bockris, J.M., Conway, B.E., White, R.E., Eds.; Springer: New York, NY, USA, 2001; Volume 34, Chapter 3, pp. 53-118.

13. Liu, Z.; Ling, X.Y.; Su, X.; Lee, J.Y. Carbon-supported Pt and PtRu nanoparticles as catalysts for a direct methanol fuel cell. J. Phys. Chem. B 2004, 108, 8234-8240.

14. Ebbesen, T.W.; Lezec, H.J.; Hiura, H.; Bennett, J.W.; Ghaemi, H.F.; Thio, T. Electrical conductivity of individual carbon nanotubes. Nature 1996, 382, 54-56.

15. Baughman, R.H.; Zakhidov, A.A.; de Heer, W.A. Carbon nanotubes - the route toward applications. Science 2002, 297, 787-792.

16. Matsumoto, T.; Komatsu, T.; Arai, K.; Yamazaki, T.; Kijima, M.; Shimizu, H.; Takasawa, Y.; Nakamura, J. Reduction of Pt usage in fuel cell electrocatalysts with carbon nanotube electrodes. Chem. Commun. 2004, 7, 840-841.

17. Yoshitake, T.; Shimakawa, Y.;. Kuroshima, S.; Kimura, H.; Ichihashi, T.; Kubo, Y.; Kasuya, D.; Takahashi, K.; Kokai, F.; Yudasaka, M.; Iijima, S. Preparation of fine platinum catalyst supported on single-wall carbon nanohorns for fuel cell application. Physica B 2002, 323, 124-126.

18. Huang, J.E.; Guo, D.J.; Yao, Y.G.; Li, H.L. High dispersion and electrocatalytic properties of platinum nanoparticles on surface-oxidized single-walled carbon nanotubes. J. Electroanal. Chem. 2005, 577, 93-97.

19. Mu, Y.; Liang, H.; Hu, J.; Jiang, L.; Wan, L. Controllable Pt nanoparticle deposition on carbon nanotubes as an anode catalyst for direct methanol fuel cells. J. Phys. Chem. B 2005, 109, 22212-22216.

20. Cansell, F.; Aymonir, C. Design of functional nanostructured materials using supercritical fluids. J. Supercrit. Fluids 2009, 47, 508-516.

21. Liu, S.M.; Han, B.X. Synthesis of carbon-nanotube composites using supercritical fluids and their potential applications. Adv. Mater. 2009, 21, 825-829.

22. Watkins, J.J.; McCarthy, T.J. Polymer/metal nanocomposite synthesis in supercritical $\mathrm{CO}_{2}$. Chem. Mater. 1995, 7, 1991-1994.

23. Lin, Y.; Cui, X.; Yen, C.; Wai, C.M. Platinum/carbon nanotube nanocomposite synthesized in supercritical fluid as electrocatalysts for low-temperature fuel cells. J. Phys. Chem. B 2005, 109, 14410-14415.

24. Saquing, C.D.; Kang, D.; Aindow, M.; Erkey, C. Investigation of the supercritical deposition of platinum nanoparticles into carbon aerogels. Microporours Mesoporours Mater. 2005, 80, 11-23.

25. Zhang, Y.; Kang, D.; Saquing, C.; Aindow, M.; Erkey, C. Supported platinum nanoparticles by supercritical deposition. Ind. Eng. Chem. Res. 2005, 44, 4161-4164. 
26. Zhang, Y.; Erkey, C. Preparation of supported metallic nanoparticles using supercritical fluids. $J$. Supercrit. Fluids 2006, 38, 252-267.

27. Bayrakceken, A.; Kitkamthorn, U.; Aindow, M.; Erkey, C. Decoration of multi-wall carbon nanotubes with platinum nanoparticles using supercritical deposition with thermodynamic control of metal loading. Scr. Mater. 2007, 56, 101-103.

28. Machino, T.; Takeuchi, W.; Kano, H.; Hiramatsu, M.; Hori, M. Synthesis of platinum nanoparticles on two-dimensional carbon nanostructures with an ultrahigh aspect ratio employing supercritical fluid chemical vapor deposition process. Appl. Phys. Express 2009, 2, 025001:1-025001:3.

29. Hiramatsu, M.; Machino, T.; Mase, K.; Hori, M.; Kano, H. Preparation of platinum nanoparticles on carbon nanostructures using metal-organic chemical fluid deposition employing supercritical carbon dioxide. J. Nanoscci. Nanotech. 2010, 10, 4023-4029.

30. Kondo, S.; Hori, M.; Yamakawa, K.; Den, S.; Kano, H.; Hiramatsu, M. Highly reliable growth process of carbon nanowalls using radical injection plasma-enhanced chemical vapor deposition. J. Vac. Sci. Technol. B 2008, 26, 1294-1300.

31. Hiramatsu, M.; Nagao, H.; Taniguchi, M.; Amano, H.; Ando, Y.; Hori, M. High-rate growth of films of dense, aligned double-walled carbon nanotubes using microwave plasma-enhanced chemical vapor deposition. Jpn. J. Appl. Phys. 2005, 44, L693-L695.

32. Hiramatsu, M.; Deguchi, T.; Nagao, H.; Hori, M. Aligned growth of single-walled and doublewalled carbon nanotube films by control of catalyst preparation. Jpn. J. Appl. Phys. 2007, 46, L303-L306.

33. Pitchon, V.; Fritz, A. The relation between surface state and reactivity in the $\mathrm{DeNO}_{\mathrm{X}}$ mechanism on platinum-based catalysts. J. Catal. 1999, 186, 64-74.

34. Ngo, T.; Brandt, L.; Williams, R.S.; Kaesz, H.D. Scanning tunneling microscopy study of platinum deposited on graphite by metalorganic chemical vapor deposition. Surf. Sci. 1993, 291, 411-417.

35. Erkey, C. Preparation of metallic supported nanoparticles and films using supercritical fluid deposition. J. Supercritical Fluids 2009, 47, 517-522.

36. Kobayashi, K.; Tanimura, M.; Nakai, H.; Yoshimura, A.; Yoshimura, H.; Kojima, K.; Tachibana, M. Nanographite domains in carbon nanowalls. J. Appl. Phys. 2007, 101, 094306:1-094306:4.

37. Kurita, S.; Yoshimura, A.; Kawamoto, H.; Uchida, T.; Kojima, K.; Tachibana, M.; MolinaMorales, P.; Nakai, H. Raman spectra of carbon nanowalls grown by plasma-enhanced chemical vapor deposition. J. Appl. Phys. 2005, 97, 104320:1-104320:5.

(C) 2010 by the authors; licensee Molecular Diversity Preservation International, Basel, Switzerland. This article is an open-access article distributed under the terms and conditions of the Creative Commons Attribution license (http://creativecommons.org/licenses/by/3.0/). 